\title{
Impaired wakefulness and rapid eye movement sleep in dopamine-deficient mice
}

\author{
Mitsuaki Kashiwagi ', Mika Kanuka ${ }^{1}$, Kaeko Tanaka' ${ }^{1}$ Masayo Fujita², Ayaka Nakai 1,3, Chika Tatsuzawa', \\ Kazuto Kobayashi ${ }^{4}$, Kazutaka Ikeda ${ }^{2}$ and Yu Hayashi ${ }^{1,5^{*}}$
}

\begin{abstract}
Despite the established roles of the dopaminergic system in promoting arousal, the effects of loss of dopamine on the patterns of sleep and wakefulness remain elusive. Here, we examined the sleep architecture of dopaminedeficient (DD) mice, which were previously developed by global knockout of tyrosine hydroxylase and its specific rescue in noradrenergic and adrenergic neurons. We found that DD mice have reduced time spent in wakefulness. Unexpectedly, DD mice also exhibited a marked reduction in the time spent in rapid eye movement (REM) sleep. The electroencephalogram power spectrum of all vigilance states in DD mice were also affected. These results support the current understanding of the critical roles of the dopaminergic system in maintaining wakefulness and also implicate its previously unknown effects on REM sleep.
\end{abstract}

Keywords: Dopamine, Mouse, NREM sleep, REM sleep

\section{Introduction}

In contrast to the rapidly growing knowledge on the anatomy of sleep-wake regulating circuits, the molecular mechanism of sleep-wake regulation remains largely unknown [1]. We focused on dopamine, a neurotransmitter that plays important roles in various brain functions including reward, motor control, and sleep-wake regulation. Psychostimulant drugs such as D-amphetamine and modafinil increase wakefulness [2]. Dopamine transporter knockout mice, in which extracellular dopamine is increased, have increased wakefulness [2]. Optogenetic or chemogenetic activation of dopaminergic neurons in the ventral tegmental area (VTA) [3, 4] or dorsal raphe [5] induces wakefulness, respectively. While the effect of activating the dopaminergic system seems well established, the effect of inactivation remains obscure. Lesion of dopaminergic ventral periaqueductal gray neurons with 6-hydroxydopamine reduces wakefulness [6].

\footnotetext{
*Correspondence: hayashi.yu.4n@kyoto-u.ac.jp

${ }^{5}$ Department of Human Health Sciences, Graduate School of Medicine, Kyoto University, Kyoto 606-8507, Japan
}

Full list of author information is available at the end of the article
Pharmacological inhibition [7] or genetic deletion [8] of the dopamine receptors also reduces wakefulness. By contrast, the effects of acute inhibition of VTA dopaminergic neurons using chemogenetics varied across studies, either reducing or having no effect on the time spent in wakefulness [3, 4]. Genetic ablation of VTA dopaminergic neurons also has apparently no effect [9]. In addition, the effect of inactivating the dopaminergic system on REM sleep remains elusive. For example, the effects of pharmacological [7] or genetic [8] intervention of dopamine receptors on REM sleep are diverse.

To directly address the effect of loss of dopamine on sleep and wakefulness, we focused on the DD mice [10]. DD mice were developed by global knockout of tyrosine hydroxylase plus its specific rescue in noradrenergic and adrenergic neurons using the dopamine beta hydroxylase promoter [10]. DD mice are hypoactive in their home cage [10]. However, their sleep architecture remained unknown. Thus, in the present study, we simultaneously recorded the electroencephalogram (EEG) and electromyogram (EMG) from DD mice to examine their sleep architecture (Additional file 1). original author(s) and the source, provide a link to the Creative Commons licence, and indicate if changes were made. The images or other third party material in this article are included in the article's Creative Commons licence, unless indicated otherwise in a credit line to the material. If material is not included in the article's Creative Commons licence and your intended use is not permitted by statutory regulation or exceeds the permitted use, you will need to obtain permission directly from the copyright holder. To view a copy of this licence, visit http://creativecommons.org/licenses/by/4.0/. The Creative Commons Public Domain Dedication waiver (http://creativeco mmons.org/publicdomain/zero/1.0/) applies to the data made available in this article, unless otherwise stated in a credit line to the data. 


\section{Results}

DD mice exhibited impaired wakefulness and REM sleep Dopamine-deficient (DD) mice were maintained with daily intraperitoneal administration of $50 \mathrm{mg} /$ $\mathrm{kg}$ L-DOPA, starting from around postnatal day 10 . Wildtype (WT) mice were administered with saline at least 3 times before EEG/EMG recording. EEG/EMG recordings were conducted $48 \mathrm{~h}$ after the last administration of $50 \mathrm{mg} / \mathrm{kg}$ L-DOPA (to DD mice) or saline (to WT mice). EEG/EMG signals were recorded for $24 \mathrm{~h}$ as previously described $[11,12]$.

DD mice exhibited reduced time spent in wakefulness and REM sleep, and increased time spent in non-REM (NREM) sleep (Fig. 1A). Wakefulness was reduced in the dark phase, whereas REM sleep was decreased in the light phase (Fig. 1A). For wakefulness and NREM sleep, DD mice exhibited reduced mean episode duration and increased episode number (Fig. 1B, C), demonstrating that these states are highly fragmented. Oppositely, for REM sleep, DD mice exhibited increased mean episode duration and reduced episode number (Fig. 1B, C). We also compared the EEG power spectrum of WT and DD mice. During wakefulness, DD mice exhibited increased EEG spectral power at $1.5-6.0 \mathrm{~Hz}$ and reduced power at $8.0-9.5 \mathrm{~Hz}$ (Fig. 1D). During NREM sleep, DD mice exhibited increased EEG spectral power at $2.5-3.0 \mathrm{~Hz}$ and reduced power at $1.5 \mathrm{~Hz}$ and $6.5-11.0 \mathrm{~Hz}$ (Fig. 1D). During REM sleep, DD mice exhibited increased EEG spectral power at 4.5-7.0 $\mathrm{Hz}$ (Fig. 1D). Thus, the EEG activity of DD mice was different from that of WT mice (Additional file 2).

\section{Discussion}

In this study, we found abnormalities in the sleep architecture of DD mice. Wakefulness was reduced and highly fragmented, indicating that DD mice cannot properly maintain wakefulness. Our observations are in line with the previously reported hypoactive phenotype of DD mice [10] and the critical roles of the dopaminergic system in maintaining wakefulness [2-9]. Importantly, loss of VTA dopaminergic neurons did not affect the time spent in wakefulness according to a viral vector-mediated ablation study [9]. Thus, dopaminergic neurons in the other areas might contribute to maintaining wakefulness.
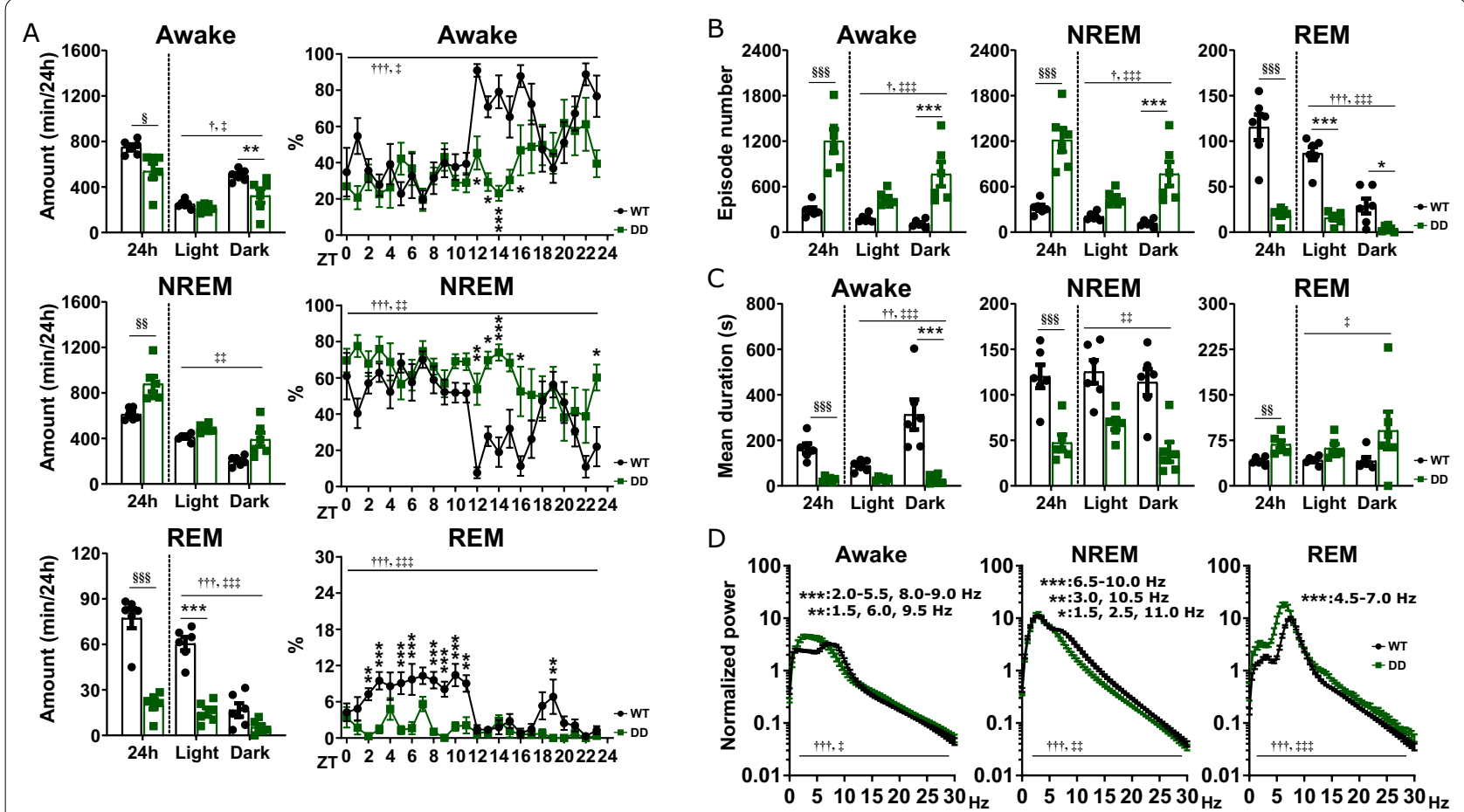

Fig. 1 Analysis of sleep architecture of DD mice. A Time spent in wakefulness, NREM sleep, and REM sleep in WT mice and DD mice. ZT zeitgeber time. B, C Episode numbers and mean durations of each vigilance state in WT mice and DD mice. D EEG power spectrum of each vigilance state in WT mice and DD mice. Frequency ranges with significant difference in the Bonferroni's test between WT mice and DD mice are described within

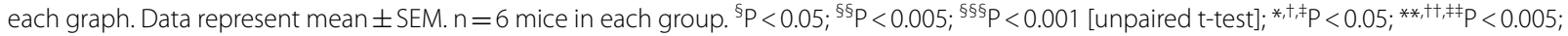

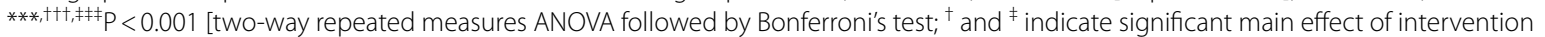
and significant interaction between intervention and time, respectively, in two-way repeated measures ANOVA, and * indicates significance in Bonferroni's test] 
Unexpectedly, we also found that REM sleep is drastically reduced in DD mice. Considering that REM sleep typically follows deep NREM sleep, reduced REM sleep might be a result of fragmented NREM sleep in DD mice. However, it should be noted that dopamine D2 receptor knockout mice also exhibit highly fragmented NREM sleep in the dark phase and yet the amount of REM sleep is unaffected [3]. Genetic activation of dopaminergic neurons in the VTA or dorsal raphe does not increase the time spent in REM sleep [3-5]. On the other hand, dopaminergic neurons in the VTA are active during REM sleep according to fiber photometry recordings [3] and the dopamine concentration in the nucleus accumbens and prefrontal cortex, where the VTA dopaminergic neurons massively project, is increased during REM sleep according to measurements by microdialysis [13]. In addition, several drugs that are considered to antagonize dopamine receptors reduce the time spent in REM sleep [7]. Thus, dopamine might contribute to the positive regulation of REM sleep through unknown mechanisms. We also found abnormalities in the EEG power spectrum of DD mice, implying that dopamine also contributes to the patterns of neuronal synchrony and/or oscillation.

One technical limitation of this study is that there is a possibility that the daily L-DOPA treatment and/ or consecutive intraperitoneal injections in DD mice might have had some effect on sleep. However, $50 \mathrm{mg} /$ $\mathrm{kg}$ L-DOPA injection does not affect the amount of each vigilance state in WT mice [14]. In addition, at the timing of EEG/EMG recordings ( $48 \mathrm{~h}$ after the last $50 \mathrm{mg} / \mathrm{kg}$ L-DOPA injection), dopamine level in the striatum of DD mice is expected to be largely depleted according to our previous study (extracellular dopamine [fmol/10 $\mathrm{min}] 24 \mathrm{~h}$ after the last $50 \mathrm{mg} /$ $\mathrm{kg}$ L-DOPA injection: WT $39.23 \pm 3.76$, DD mice $0.62 \pm 0.12 ; 72 \mathrm{~h}$ after the last $50 \mathrm{mg} / \mathrm{kg} \mathrm{L}$-DOPA injection: WT $43.55 \pm 4.80$, undetectable in DD mice) [15]. Another technical issue is that the transgenic expression of tyrosine hydroxylase in DD mice might affect the level of other neurotransmitters such as noradrenaline, although we previously confirmed that noradrenaline level in the striatum of DD mice was unaffected (extracellular noradrenaline [fmol/10 min] $72 \mathrm{~h}$ after the last $50 \mathrm{mg} / \mathrm{kg} \mathrm{L}$-DOPA injection: WT $0.51 \pm 0.07$, DD $0.67 \pm 0.09)[15]$.

In summary, by direct measurement of EEG/EMG and analysis of sleep architecture in DD mice, this study further supports the current understanding of the critical roles of dopaminergic system in maintaining wakefulness and implicates its overlooked effects on the positive regulation of REM sleep.
Abbreviations

REM sleep: Rapid eye movement sleep; NREM sleep: Non-REM sleep; DD mouse: Dopamine-deficient mouse; EEG: Electroencephalogram; EMG: Electromyogram; VTA: Ventral tegmental area; ZT: Zeitgeber time.

\section{Supplementary Information}

The online version contains supplementary material available at https://doi. org/10.1186/s13041-021-00879-3.

Additional file 1. Materials and methods.

Additional file 2. Raw data presented in this study.

\section{Authors' contributions}

MKas, MKan, KT, MF, AN, CT, KK, Kl, and YH designed and performed the experiments. MKas analyzed the data. MKas and $\mathrm{YH}$ wrote the manuscript with inputs from MF and KI. All authors read and approved the final manuscript.

\section{Funding}

This work was supported by the JSPS KAKENHI under Grant Numbers JP20K22674 and JP 21K15179 (to M. Kashiwagi), JP20H03353 (to Y.H.), and JP16H06276 (AdAMS) (to K.I.); the MEXT KAKENHI under Grant Numbers JP21H00414 and JP21H00199 (to Y.H.); AMED under Grant Numbers JP19gm1110008 and JP21 Wm0425018 (to Y.H.); and the MEXT WPI program (to Y.H.).

\section{Availability of data and materials}

All data are available upon reasonable request to the corresponding author.

\section{Declarations}

\section{Ethics approval and consent to participate}

All required animal ethics approvals have been obtained for this research.

\section{Consent for publication}

This study does not contain any individual person's data.

\section{Competing interests}

The authors declare no competing interests.

\section{Author details}

${ }^{1}$ International Institute for Integrative Sleep Medicine (WPI-IIIS), University of Tsukuba, Tsukuba 305-8575, Japan. ${ }^{2}$ Addictive Substance Project, Tokyo Metropolitan Institute of Medical Science, Setagaya-ku, Tokyo 156-8506, Japan. ${ }^{3}$ Doctoral Programs in Neuroscience, Degree Programs in Comprehensive Human Sciences, University of Tsukuba, Tsukuba 305-8575, Japan. ${ }^{4}$ Department of Molecular Genetics, Institute of Biomedical Sciences, Fukushima Medical University, Fukushima 960-1295, Japan. ${ }^{5}$ Department of Human Health Sciences, Graduate School of Medicine, Kyoto University, Kyoto 606-8507, Japan.

Received: 26 August 2021 Accepted: 8 November 2021

Published online: 18 November 2021

\section{References}

1. Kashiwagi M, Hayashi Y. The existence of two states of sleep as a common trait in various animals and its molecular and neuronal mechanisms. Curr Opin Physiol. 2020;15:197-202.

2. Wisor JP, Nishino S, Sora I, Uhl GH, Mignot E, Edgar DM. Dopaminergic role in stimulant-induced wakefulness. J Neurosci. 2001;21:1787-94.

3. Eban-Rothschild A, Rothschild G, Giardino WJ, Jones JR, De Lecea L. VTA dopaminergic neurons regulate ethologically relevant sleep-wake behaviors. Nat Neurosci. 2016;19:1356-66.

4. Oishi Y, Suzuki Y, Takahashi K, Yonezawa T, Kanda T, Takata Y, Cherasse Y, Lazarus M. Activation of ventral tegmental area dopamine neurons produces wakefulness through dopamine D 2-like receptors in mice. Brain Struct Funct. 2017:222:2907-15. 
5. Cho JR, Treweek JB, Robinson JE, Xiao C, Bremner LR, Greenbaum A, Gradinaru V. Dorsal raphe dopamine neurons modulate arousal and promote wakefulness by salient stimuli. Neuron. 2017;94:1205-1219.e8.

6. Lu J, Jhou TC, Saper CB. Identification of wake-active dopaminergic neurons in the ventral periaqueductal gray matter. J Neurosci. 2006;26:193-202.

7. Ongini E, Bonizzoni E, Ferri N, Milani S, Trampus M. Differential effects of dopamine D-1 and D-2 receptor antagonist antipsychotics on sleepwake patterns in the rat. J Pharmacol Exp Ther. 1993;266:726-31.

8. Qu WM, Xu XH, Yan MM, Wang YQ, Urade Y, Huang ZL. Essential role of dopamine $\mathrm{d} 2$ receptor in the maintenance of wakefulness, but not in homeostatic regulation of sleep, in mice. J Neurosci. 2010;30:4382-9.

9. Takata Y, Oishi Y, Zhou X-Z, Hasegawa E, Takahashi K, Cherasse Y, Sakurai T, Lazarus M. Sleep and wakefulness are controlled by ventral medial midbrain/pons GABAergic neurons in mice. J Neurosci. 2018;38:10080-92.

10. Fujita M, Hagino Y, Takeda T, Kasai S, Tanaka M, Takamatsu Y, Kobayashi K, Ikeda K. Light/dark phase-dependent spontaneous activity is maintained in dopamine-deficient mice. Mol Brain. 2017;10:1-10.

11. Hayashi Y, Kashiwagi M, Yasuda K, Ando R, Kanuka M, Sakai K, Itohara S. Cells of a common developmental origin regulate REM/non-REM sleep and wakefulness in mice. Science. 2015;350:957-61.
12. Kashiwagi M, Kanuka M, Tatsuzawa C, Suzuki H, Morita M, Tanaka K, Kawano T, Shin JW, Suzuki H, Itohara S, et al. Widely distributed neurotensinergic neurons in the brainstem regulate NREM sleep in mice. Curr Biol. 2020;30:1002-1010.e4.

13. Léna I, Parrot S, Deschaux O, Muffat-Joly S, Sauvinet V, Renaud B, SuaudChagny MF, Gottesmann C. Variations in extracellular levels of dopamine, noradrenaline, glutamate, and aspartate across the sleep-wake cycle in the medial prefrontal cortex and nucleus accumbens of freely moving rats. J Neurosci Res. 2005:81:891-9.

14. Laloux C, Derambure P, Houdayer E, Jacquesson JM, Bordet R, Destée A, Monaca C. Effect of dopaminergic substances on sleep/wakefulness in saline- and MPTP-treated mice. J Sleep Res. 2008;17:101-10.

15. Hagino Y, Kasai S, Fujita M, Setogawa S, Yamaura H, Yanagihara D, Hashimoto M, Kobayashi K, Meltzer HY, Ikeda K. Involvement of cholinergic system in hyperactivity in dopamine-deficient mice. Neuropsychopharmacology. 2015;40:1141-50.

\section{Publisher's Note}

Springer Nature remains neutral with regard to jurisdictional claims in published maps and institutional affiliations.
Ready to submit your research? Choose BMC and benefit from:

- fast, convenient online submission

- thorough peer review by experienced researchers in your field

- rapid publication on acceptance

- support for research data, including large and complex data types

- gold Open Access which fosters wider collaboration and increased citations

- maximum visibility for your research: over $100 \mathrm{M}$ website views per year

At BMC, research is always in progress.

Learn more biomedcentral.com/submissions 